\title{
Etude sur le salaire horaire
}

\section{Beatrice Brunnera, Esther Kraft ${ }^{b}$}

a WIG/ZHAW, responsable suppléante en recherche économique sur les soins, Winterthour

${ }^{b} \mathrm{FMH}$, cheffe de la division Données, démographie et qualité, Berne

\section{Introduction}

La constante augmentation des coûts de la santé et des primes d'assurance-maladie a accru le besoin de transparence sur le revenu des médecins. C'est la raison pour laquelle différentes études et enquêtes se sont penchées sur cette question en s'appuyant sur diverses sources de données [1-3]. Pour la FMH, la transparence et la fiabilité des données sont les fondements d'une discussion sur le revenu des médecin objective, basée sur des faits et axée sur la recherche de solutions.

\section{Diverses études et enquêtes}

Le 10 avril 2018, l'Office fédéral de la statistique (OFS) a publié les résultats du premier relevé des données structurelles des cabinets médicaux et des centres ambulatoires (MAS) de 2015 [2]. Effectué sur la base de l'article 23 de la loi sur l'assurance-maladie (LAMal) et en application de la loi sur la statistique fédérale (LSF), ce relevé a servi uniquement à des fins statistiques. Sur les 13971 cabinets médicaux et centres ambulatoires consultés, la moitié a fourni des données exhaustives. Il a ainsi été possible de procéder pour la première fois à une analyse approfondie de la situation financière des cabinets médicaux et des centres ambulatoires en Suisse. L'analyse révèle un chiffre d'affaires global moyen de 545000 CHF pour les médecins en cabinet individuel avec un total de dépenses de $390000 \mathrm{CHF}$ en moyenne. Il en résulte un revenu net de $155000 \mathrm{CHF}$ en 2015 , dont $89 \%$ sont financés par l'assurance obligatoire des soins. Pour les cabinets de groupe, en revanche, il n'a pas été possible de calculer le revenu de chaque médecin [4].

A la fin d'octobre 2018, l'Office fédéral de la santé publique a publié une étude du bureau BASS sur le revenu des médecins en Suisse [3]. Les indications sur le revenu s'appuient sur les données AVS des caisses de compensation pour l'année 2014, qui prennent en compte la totalité des médecins porteurs d'un titre de spécialiste. Selon l'étude du bureau BASS, le revenu annuel brut moyen s'élevait à $233791 \mathrm{CHF}$ et le revenu médian à 190577 CHF en 2014. A ce propos, il convient de noter que ces chiffres concernent les revenus des médecins du secteur ambulatoire et hospitalier et qu'ils ne tiennent pas compte d'environ un cinquième du corps médical, à savoir les médecins-assistants, dont le revenu annuel est d'environ $100000 \mathrm{CHF}$ pour 56 heures de travail hebdomadaire. De plus, cette étude sur le revenu date de 2014, donc avant les réductions tarifaires imposées par le Conseil fédéral, et ne permet pas de visualiser la part du revenu des médecins financée par l'assurance obligatoire des soins.

Ces deux études fournissent des résultats importants sur le revenu annuel des médecins en Suisse, sans tenir compte cependant du nombre d'heures réellement travaillées. Il est donc impossible de déterminer si une éventuelle différence dans le revenu annuel est liée au salaire horaire ou à un volume de travail plus important. Les comparaisons salariales significatives ne sont possibles que sur la base des salaires horaires, or il n'existe jusqu'à présent aucune étude de ce type pour les médecins en Suisse.

Dans ce contexte, la FMH a mandaté la Haute école des sciences appliquées de Zurich (ZHAW) pour calculer le salaire horaire des médecins et le comparer à celui d'autres groupes professionnels de niveau universitaire.

\section{Etude sur le salaire horaire - comparer ce qui est comparable}

L'objectif de l'étude est de calculer le salaire horaire des médecins en Suisse en tenant compte du volume effectif de travail, de la part de cotisations sociales et du statut professionnel (salarié ou indépendant) [5]. Le salaire horaire des autres groupes professionnels universitaires a été déterminé selon la même base de calcul pour ensuite être comparé à celui des médecins. L'étude s'appuie sur l'enquête suisse sur la population active (ESPA), réalisée chaque année par l'OFS auprès d'environ 65000 personnes, dont près de $63 \%$ sont en activité (le reste est soit en formation continue, au chômage ou à la retraite) [6]. L’ESPA comprend entre autres des informations détaillées sur la profession, le salaire, le taux d'occupation, le statut professionnel et les heures effectives de travail, ce qui fait qu'elle est actuellement la seule source de données pour calculer le salaire horaire des différents groupes professionnels. Selon l'OFS, elle comporte un point faible, à savoir que sa représentativité professionnelle n'est pas garantie. 


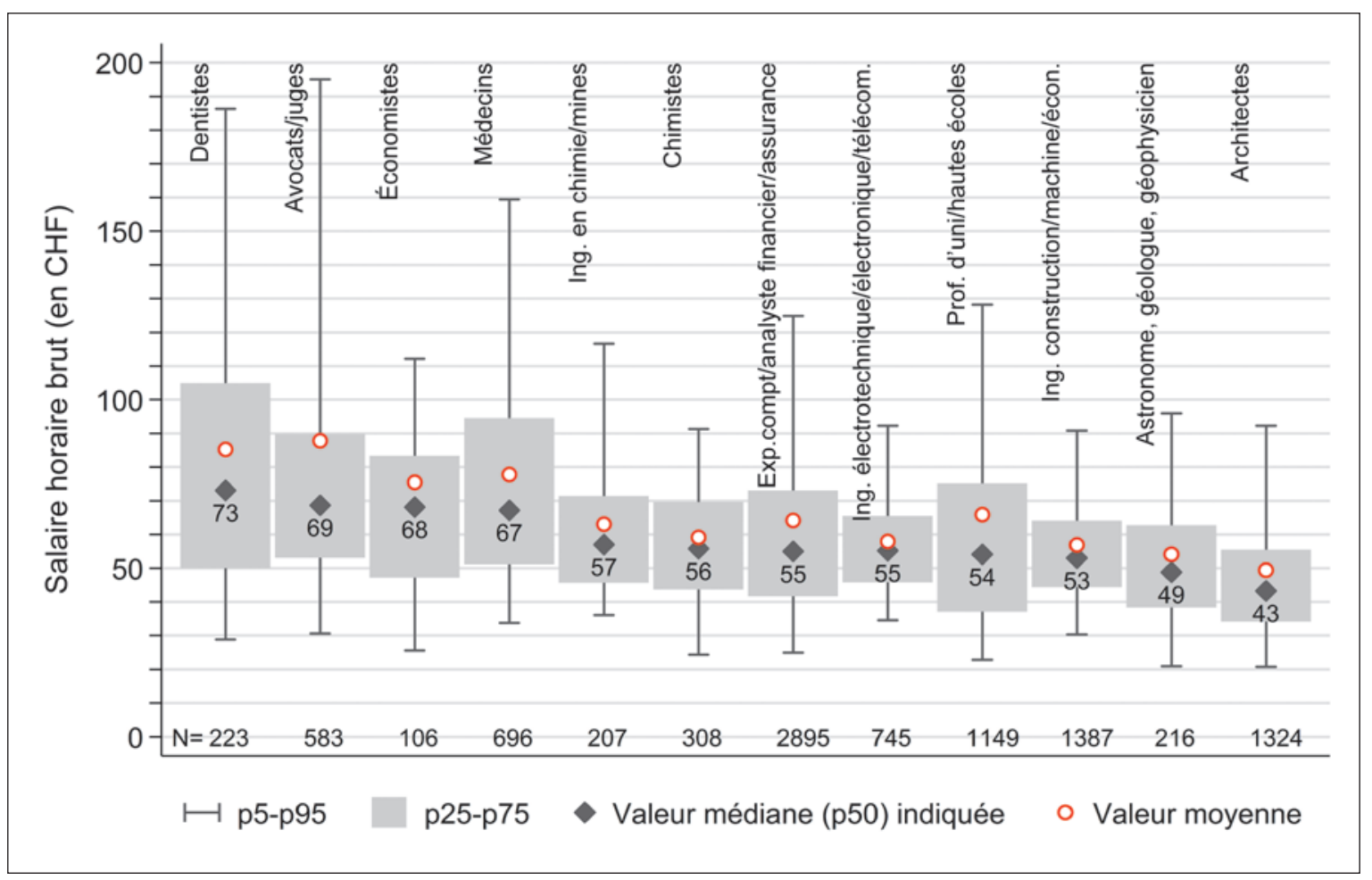

Figure 1: Salaire horaire standardisé des différentes professions (2014-2017).

\section{Résultats}

Au cours de la période d'observation de 2014 à 2017, le nombre de médecins en exercice clairement identifiables inclus dans l'enquête a fluctué entre 159 et 192 par an. Afin de réduire les fluctuations aléatoires liées au nombre relativement bas de cas observés, les chiffres des années 2014-2017 ont été réunis.

Durant ces quatre années, le salaire horaire brut médian des médecins inclus dans l'enquête était de 67 CHF (la moyenne étant de $78 \mathrm{CHF}$ ), le classant ainsi au quatrième rang des groupes professionnels examinés. La première place revient aux dentistes avec un revenu médian de $73 \mathrm{CHF} / \mathrm{h}$, suivis des avocats et des juges $(69 \mathrm{CHF} / \mathrm{h})$ et des économistes $(68 \mathrm{CHF} / \mathrm{h})$.

Le résultat est différent lorsque c'est le revenu annuel brut standardisé en équivalents temps plein qui est comparé, à la place du salaire horaire. Avec un revenu médian de 163000 CHF, les médecins se placent alors au premier rang, ce qui signifie que les revenus annuels relativement élevés des médecins par rapport aux autres groupes professionnels sont en partie dus à des horaires de travail supérieurs à la moyenne.

Les auteurs de l'étude calculent les revenus annuels standardisés et non standardisés afin de permettre la comparaison avec d'autres études. Le revenu annuel brut moyen se monte à $155329 \mathrm{CHF}$ et le revenu brut médian à $140000 \mathrm{CHF}$. Comme la pondération des temps pleins et des temps partiels est la même, les valeurs du revenu non standardisées sont en moyenne inférieures à celles des revenus standardisés.

La distinction selon le statut professionnel montre qu'avec un salaire horaire médian de $75 \mathrm{CHF}$, les médecins indépendants gagnent 19\% de plus que les médecins salariés $(63 \mathrm{CHF} / \mathrm{h})$.

\section{Conclusions et perspectives}

Cette étude innove dans la mesure où elle calcule et compare le salaire horaire des médecins avec d'autres groupes professionnels en Suisse. Il est ainsi possible de comparer des groupes professionnels équivalents au sein d'un collectif clairement défini sur le plan statistique (ESPA).

A l'avenir, la discussion sur les revenus des médecins devrait se baser sur un salaire horaire et il faudrait renoncer à observer les écarts statistiques de manière sélective. La comparaison des salaires horaires reflète de manière transparente le taux d'occupation réel et permet une discussion objective, basée sur des faits et axée sur la recherche de solutions. 


\section{Références}

1 Künzi K, Strub S. Revenus des médecins indépendants: analyse des données 2009 de Medisuisse. Bull Med Suisses. 2012;93(38):1371-5.

2 MAS, Données structurelles des cabinets médicaux et des centres ambulatoires (MAS). OFS; 2015: https://www.bfs.admin.ch/bfs/fr/ home/statistiques/sante/enquetes/sdapaz.html

3 Künzi K, Morger M. Revenus, prestations de l'assurance obligatoire des soins et situation de l'emploi des médecins 2009-2014. Berne: Bureau d'études de politique du travail et de politique sociale BASS; 2018.

4 Kraft E. La Confédération et la FMH arrivent au même résultat. Bull Med Suisses. 2018;99(43):1480-1.

5 Brunner B., Stundenlohnstudie: Vergleich zwischen Ärzten und anderen Berufsgruppen auf Basis der SAKE. ZHAW, Winterthurer Institut für Gesundheitsökonomie, 2019

6 Enquête suisse sur la population active (ESPA). OFS; 1996-2017: https://www.bfs.admin.ch/bfs/fr/home/statistiques/travailremuneration/enquetes/espa.html 